Tuy niên siêu âm không phải là phương tiện chẩn đoán hình ảnh duy nhất, trong những trường hợp thương tổn phối hợp khó chẩn đoán, đôi khi phải kết hợp với các phương tiện khác như xét nghiệm huyết học, $X$ quang, CT scan, MRI, chọc dò ổ bụng, nội soi ổ bụng chẩn đoán...
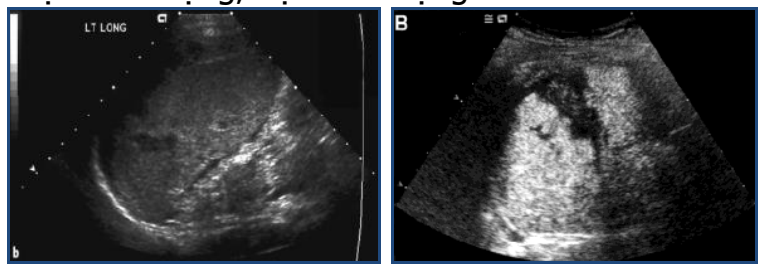

\section{Hình ảnh vỡ lách}

\section{KẾT LUÂN}

Chấn thương và vết thương bụng là cấp cứu ngoại khoa. Chẩn đoán sớm, thái độ xử lý thích hợp giúp giảm tỷ lệ tử vong.

Siêu âm đánh giá tập trung thương tổn là phương pháp chẩn đoán được lựa chơn đầu tiên giúp khảo sát thương tổn tạng trong ổ bụng.

Độ nhạy, độ chính xác của siêu âm trong chẩn đoán chấn thương và vết thương bụng khá cao.

\section{TÀI LIÊU THAM KHẢO}

1. Phạm Hòa Anh (2000), Giá trị chẩn đoán của siêu âm trong chân thương bunng kín. Luận văn Thạc sĩ y khoa. Trường Đại học Y Y dược TP.HCM.

2. Boulanger B.R (1996), Emergent abdominal sonography as creening test in a new diagnosis algorithm for blunt trauma, J Trauma Jun, 40(6): 867-74.

3. Lê Tư Hoàng (2004), Chẩn đoán và xử trí chấn thương bung kín tai Bênh viên Việt Đức 20012003, Ngoại khoa, số 5, tr 11-17.

4. Ingerman J.F. (1996), Emergency phýsicians use ultrasonography in blunt abdominal trauma. Acad Emerg Med. 3(10), p 931-937.

5. Huỳnh Trung Lập (2007), Giá trị của siêu âm trong chẩn đoán chấn thương bụng kín. Luận văn Chuyên khoa II. Trường Đai hoc Y $Y$ dước TP. HCCM.

6. Lui M. (1993), Prospective comparison of diagnosis peritoneal lavage, computed tomography scanning and ultrasonography for the diagnosis of blunt abdominal trauma. J Trauma Aug, 35(2): 267-70.

7. Mc Kenney G. (1998), Can surgeons evaluate emergency ultrasound scan for blunt abdominal trauma. J Trauma Nov, 44(4): 649-53.

8. Michihiro S. (2004), Reevaluation of ultrasonography for solid "organ injury in blunt abdominal trauma. Acad Emerg Med. 9(10), p 68-70.

\title{
ĐÁNH GIÁ VI KẼ CỦA CLEARFIL AP-X COMPOSITE KHI SỬ DỤNG HAI KỸ THUẬT ĐẶT CHẤT DÁN KHÁC NHAU
}

\section{TÓM TẮT}

Mục tiêu: Đánh giá khả năng làm giảm vi kẽ của hệ thổng dán Single Bond Universal Adhesive khi áp dụng kỹ thuật đặt 2 lớp keo dán chiếu đèn 1 lần và đắt 2 lớp keo dán chiếu đèn riêng từng lớp. Phương pháp nghiên cứu: Nghiên cứu thử nghiệm trong labo (in vitro) trên 30 răng cối nhỏ hàm dưới vĩnh viến của người trưởng thành, chia ngẫu nhiên thành 2 nhóm với kỹ thuât sử dung hệ thống dán Single Bond Universal Adhesive đặt 2 lớp và chiếu đèn một lần (nhóm 1), so với kỹ thuật đặt 2 lớp bond, chiếu đèn riêng cho từng lớp. Kết quả: Vi kẽ đến thành trục được quan sát thấy ở cà hai nhóm. Tuy nhiên số lượng mẫu có vi kẽ đến thành trục ở nhóm chiếu đèn tựng lớp ít hơn so với nhóm chiểu đèn 1 lần sau khi đă̆t 2 lớp chất dán (2 mẫu độ 3 ở nhóm II và 9 mẫu đổ 3 ở nhóm I). Mức độ vi kẽ ở thành nướu của phục hồi xoang II ở nhóm chiếu đèn từng lớp là ít hơn có ý nghĩa so với nhóm chiếu đèn 1 lân sau khi đặt 2 lớp

\section{*Đại học Y Dước TP HCM}

Chịu trách nhiệm chính: Nguyễn Thu Thủy

Email: ntthuy@ump.edu.vn

Ngày nhận bài: 5/3/2021

Ngày phản biện khoa học: 6/4/2021

Ngày duyệt bài: 3/5/2021

\section{Nguyễn Thu Thủy*}

chất dán $(p<0,05)$. Kết luận: Kỹ thuật đặt hai lớp chất dán và chiếu đèn cho từng lớp có thể là một biên pháp giúp khắc phục vi kẽ, một trong những thách thức lớn của phục hồi composite.

Tư khóa: Hệ thống dán Bonding, phương pháp keo dán chiếu đèn, vi kẽ.

\section{SUMMARY}

EVALUATE THE MICROLEAKAGE OF CLEARFIL AP-X COMPOSITE USING TWO DIFFERENT ADHESIVE TEACHNIQUES

Objectives: To evaluate the ability of the Single Bond Universal Adhesive system to reduce microleakage when applying 2 coats - one cure and 2 coats - two separate cures teachnique. Methods: Experimental study in the lab (in vitro) on 30 permanent lower premolars, randomly divided into 2 groups with the technique using the Single Bond Universal Adhesive system with 2 coats - one cure (group 1) and 2 coats - two separate cures (group 2). Results: Axial microleakages were observed in both groups. However, the number of samples with axial microleakages in the group of curing each layer was less than that of the one-cure group after placing 2 layers of adhesive ( 2 samples of degree 3 in group II and 9 samples of degree 3 in group I). The degree of microleakages in the gigival wall of cavities in the two- 
cure group was significantly less than that of the onecure group $(p<0.05)$. Conclusion:The technique of applying two layers of adhesive and curing each layer can be a remedy to help overcome microleakage, one of the major challenges of composite recovery.

Key words: Bonding system, light curing method, microleakage.

\section{I. ĐĂT VẤN ĐỀ}

Composites nha khoa được sử dụng rộng rãi nhờ tính thẩm mỹ cao, không chứa thủy ngân và tương hợp sinh học tốt. Tuy nhiên, phục hồi trực tiếp bằng composites có thể có những yếu tố không thuận lợi như đổi màu phục hồi, sâu răng tái phát, và nhạy cảm

Sau trám. Đây là kết quả của sự co trùng hợp và là nguyên nhân hình thành vi kẽ giữa composites resin với các thành của xoang trám. Phần vi kẽ ở thành nướu có thể chiếm từ 1,67\% đến $5,68 \%$ tổng thể tích phục hồi và dịch trong khoang miệng hay vi khuẩn có thể lắp đầy những vi kẽ này [1].

Nhiêu nghiên cứu đã chỉ ra khả năng dính của resin composites vào các thành xoang không chỉ phụ thuộc vào lực dán lên men hay ngà, mà còn biảnh hưởng bởi nhiều yếu tố khác như ngẫu lực co của resin, yếu tố $C$, phương pháp trám và mức độ trùng hợp.

Hiện nay, việc sử dụng composite lỏng và các chất dán thế hệ mới đã trở nên khá phổ biến. Tuy nhiên, sự dán của composite lên các thành xoang cũng như việc loại trừ vi kẽ vẫn còn là môt thách thức lớn.

Hệ thống dán đóng vai trò quan trọng góp phần cũng cố lớp lai và ghép resin vào trong ống ngà mở. Nhiều nghiên cứu được thực hiện nhằm tìm ra các phương pháp gia tăng hiệu quá dán của vật liệu. Nhiều nghiên cứu cho thấy hệ thống dán tự xoi mòn loại một lọ có độ bền dán tương đối thấp hơn so với hệ thống dán tự xoi mòn hai lọ cũng như hệ thống dán xoi mòn và rửa. Nguyên nhân chủ yếu do lớp chất dán quá mỏng và bịức chế trùng hợp bởi oxy không khí. Meer-beek và cộng sựđề nghị dùng cọ để quét mỏng lớp keo hớn là dùng hơi để trải mỏng, việc này là để tránh làm mỏng lớp keo tới mức bi không khí chiếm phần lớn lớp keo [6]. Theo Ruggeberg và Margeson, $15 \mu \mathrm{m}$ keo dán trên bề mặt không thể trùng hợp vì bịức chế bởi oxi. Do đó, bất cứ lớp chất dán mỏng hơn $15 \mu \mathrm{m}$ không thể trùng hợp và ngăn chặn hình thành lớp kết dính, dẫn đển hình thành vì kẽ [4]. Platt và cộng sự cho rằng độ bền dán được cải thiện khi sử dụng 2 lớp keo dán so với 1 lớp keo dánđối với hệ thống dán tự xoi mòn [2]. S.Deliperi và cộng sự (2004) cho rằng việc sử dụng kỹ thuật đặt 2 lớp keo dán và chiếu đèn từng lớp giúp đảm bảo đủđộ dày của lớp keo dán và cải thiện độ bền dán của phục hồi vào lên mô răng [5].

Mục tiêu nghiên cứu: Đánh giá khả năng làm giảm vi kẽ của hệ thống dán Single Bond Universal Adhesive khi được đặt với 2 kỹ thuật khác nhau.

\section{II. ĐỐI TƯỢNG VÀ PHƯƠNG PHÁP NGHIÊN CỨU}

2.1. Thiết kế nghiên cứu. Nghiên cứuthử nghiệm trong labo (in vitro).

2.2. Đối tượng nghiên cứu. Nghiên cứu được thực hiện trên 30 răng cối nhỏ hàm dưới vĩnh viễn của người trưởng thành, nhổ do yêu cầu chỉnh hình răng mặt.

Tiêu chuẩn chọn mẫu: Thân răng nguyên vẹn, không có tổn thương hay sang thương nào trên răng. Kích thước thân răng tương đương nhau. Răng không sâu, không có phục hồi nào.

2.3. Phương pháp tiến hành. Răng được làm sạch bằng dụng cụ cạo vôi tay, đánh bóng bằng đài cao su với bột pumice không fluor, ngâm bảo quản trong nước muối sinh lý $0,9 \%$ ớ nhiệt độ phòng.

Sửa soạn lồ trám loại II dạng hộp ở mặt xa với kích thước ngoài-trong $3 \mathrm{~mm}$, gần- xa $2 \mathrm{~mm}$, nhai-nướu $5 \mathrm{~mm}$, thành nướu đặt trên đường nối men-xi măng tối thiểu $1 \mathrm{~mm}$. Sau khi tạo xoang, đánh số răng từ 1-30, chia ngẫu nhiên thành 2 nhóm, mỗi nhóm 15.

\section{Thực hiện phục hồi}

Bước 1: Thoa keo dán

- Nhóm 1: bôi 2 lớp bond, chiếu đèn 1 lần, thời gian chiếu đèn 10 giây.

- Nhóm 2: bôi 2 lớp bond, chiếu đèn riêng cho từng lớp, thời gian chiếu đèn mỗi lớp 10 giây.

Bước 2: Đă̆t composites: Đặt composites từng lớp không quá $2 \mathrm{~mm}$ theo chiều nhai nướu, chiếu đèn riêng cho mỗi lớp.

Bước 3: Đánh bóng miếng trám: Đánh bóng bằng đĩa hoặc mũi đánh bóng silicon.

Chu trình nhiệt: 100 chu trình nhiệt giữa hai mức nhiêt độ là $55^{\circ} \mathrm{C} \pm 1^{\circ} \mathrm{C}$ và $5^{\circ} \mathrm{C} \pm 1^{\circ} \mathrm{C}$. Thời gian ngưng tại mỗi điểm nhiệt là 25 giây và thời gian chuyển đổi là 5 giây.

Ngâm mẫu vào dung dịch xanh methylene $2 \%$ trong vòng 24 giờ. Sau khi lấy ra khỏi dung dịch thuốc nhuộm, rửa sạch dưới vòi nước trong 10 phút, làm sạch lại bằng bột pumice để loại bỏ dung dịch nhuộm trên bề mặt.Bao quanh răng bằng khối nhựa tự cứng trong suốt, cắt đôi răng theo chiều gần-xa qua giữa miếng trám bằng đĩa cắt kim cương tốc độ chậm có nước làm mát. 
Quan sát dưới kính hiển vi nổi, độ phóng đại 30 lần. Đánh giá độ thâm nhập của dung dịch nhuộm ở thành nướu của phục hồi theo thang đánh giá (Ferrari và cộng sự, 1996) như sau:

0: không có sự thâm nhập của chất nhuộm

1: sự thâm nhập của chất nhuộm không quá đường nối men-ngà

2: sự thâm nhập chất nhuộm quá đường nối men ngà nhưng chưa đến thành trục

3: sự thâm nhập của chất nhuộm đến thành trục

\section{KẾT QUẢ NGHIÊN CỨU}

Nhóm đặt keo dán hai lớp và chiếu đèn sau cùng phần lớn có vi kẽđến thành trục ( 9 trên 15 mẫu), không ghi nhận mẫu không có sự thâm nhập phẩm nhuộm cũng như không ghi nhận mấu sự thâm nhập của phẩm nhuộm chiở lớp men. Giá trị trung bình về mức độ thâm nhập vi kẽ của nhóm là 2,6.

Nhóm đă̆t keo dán hai lớp và chiếu đèn mỗi lớp, phần lớn vi kẽđến ngà nhưng chưa đến thành trục, có 3 mẫu ghi nhận sự thâm nhập của phẩm nhuộm khu trú ở men, và không ghi nhận mẫu không có sự thâm nhập phẩm nhuộm. Giá trị trung bình về mức độ thâm nhập vi kẽ của nhóm là 1,9 .

Số lương mẫu không có, có vi kẽ ít hoăc trung bình (mức độ 0,1 và 2 ) ở nhóm chiếu đèn từng lớp $(86,68 \%)$ nhiều hơn so với nhóm chiếu đèn sau cùng $(40 \%)$ (Bảng 1$)$.

Bảng 1. Số lượng và tỷ lệ vi kẽ của hai nhóm

\begin{tabular}{|c|c|c|c|c|c|}
\hline & \multicolumn{4}{|c|}{ Tình trạng vi kẽ } & \multirow{3}{*}{ Tổng (\%) } \\
\hline & \multicolumn{2}{|c|}{$\begin{array}{l}\text { Không có, có vi kẽ ít hoăc } \\
\text { trung bình (mức độ } 0,1 \text { và 2) }\end{array}$} & \multicolumn{2}{|c|}{$\begin{array}{c}\text { Có nhiều vi kẽ } \\
\text { (mức độ 3) }\end{array}$} & \\
\hline & Số lượng & Tỷ lệ (\%) & Số lượng & Tỷ lệ & \\
\hline Nhóm 1 & 6 & $40 \%$ & 9 & $60 \%$ & $15(100 \%)$ \\
\hline Nhóm 2 & 13 & $86,67 \%$ & 2 & $13,33 \%$ & $15(100 \%)$ \\
\hline Tống & 19 & $63,33 \%$ & 11 & $36,67 \%$ & $30(100 \%)$ \\
\hline
\end{tabular}

Sự khác biệt về mức độ vi kẽ không, cóít hoăc trung bình so với mức độ có nhiều vi kẽ giữa nhóm chiếu đèn từng lớp và chiểu đèn sau cùng là cóý nghĩa thống kê $(p=0,008<0,05)$.

\section{BÀN LUÂN}

Cả 2 nhóm đều quan sát thấy có sự hiện của vi kẽở thành nướu của phục hồi. Nhóm đặt hai lớp chất dán và chiếu đèn một lần sau đó có $100 \%$ mẫu có vi kẽ đến ngà hay đến thành trục trong khi nhóm đặt 2 lớp chất dán và chiếu đèn từng lớp có $20 \%$ mẫu có mức độ vi kẽ chỉ ở lớp men. Giá trị trung bình về mức độ vi kẽ của nhóm đặt hai lớp chất dán và chiếu đèn một lần sau đó $(2,6)$ cao hơn so với nhóm chiếu đèn từng lớp $(1,9)$. Sự khác biệt này là có ý nghĩa theo phép kiểm phi tham số Mann-Whitney $(p=0,04<0,05)$. Khi đánh giá về mối liên hệ giữa mức độ vi kẽ và phương pháp đặt chất dán, ta nhận thấy: sự khác biệt về mức độ không có, có vi kẽ ít hay trung bình (mức độ 0,1 hoặc 2) so với mức độ có nhiều vi kẽ (mức độ 3 ) giữa hai là có ý nghĩa thống kê $(p=0,008<0,05)$ theo phép kiểm chi bình phương.

Nagpal và cộng sự (2015) đã nghiên cứu về sự ảnh hưởng cửa kĩ thuật đặt hai lớp dán của hệ thống dán tự xoi mòn, tác giả cho rằng việc đặt hai lớp hệ thống dán tự xoi mòn giúp cải thiện tính dán do tăng khả năng xoi mòn các tinh thể hydroxyapatite. Khả năng xoi mòn của lớp chất dán đâuu tiên nhanh chóng mất tác dụng khi tiếp xúc với mô men hoặc mô ngà, vì vậy lớp thứ hai có tác dụng bổ sung các đoạn mồi có tính acid, cải thiện tính dán [3].

Simone Deliperi và cộng sự (2004) báo cáo về kết quả của kỹ thuật đặt hai lớp keo dán và chiếu đèn từng lớp cho keo dán Prime $\&$ Bond NT, vi kẽ của nhóm này khác biệt không có ý nghĩa thống kê so với nhóm đặt hai lớp dán và chiếu đèn sau cùng. Tác giả cũng chỉ ra một nghiên cứu tương tự được thực hiện bởi cộng sự của ông đối với composite Pyramid cho thây kết quả của nhóm chiếu đèn từng lớp là ít vi kẽ hơn có ý nghĩa so với nhóm chiếu đèn một lần sau cùng. Tác giả cho rằng việc sử dụng nhiều lớp chất dán có tác dụng đảm bảo loại trừ được sự hình thành một lớp dán quá mỏng, bị ức chế bởi oxy và làm giảm vi kẽ. Tác giả cho rằng kết quả sự khác biệt một phần vị ảnh hưởng bởi loại composite sử dụng để phục hồi [5].

\section{KẾT LUẬN}

Việc phục hồi xoang II composite, sử dụng kỹ thuật đặt hai lớp chất dán và chiếu đèn từng lớp hay đặt hai lớp chất dán và chiếu đèn một lần đều quan sát được vi kẽ ở thành nướu của phục hồi. Vi kẽ ở thành nướu của phục hồi xoang II sử dụng composite Clearfil - APX với kỹ thuật đặt hai lớp chất dán, chiếu đèn từng lớp ít hơn có ý nghĩa thống kê so với kỹ thuật đặt hai lớp chất dán và chiếu đèn một lần. Mức độ vi kẽ ở thành 
nướu của phục hồi xoang II sử dụng composite Clearfil - APX có liên quan đến phương pháp đặt chất dán. Sự khác biệt về vi kẽ của hai kỹ thuật còn phụ thuộc vào loại Composite sử dụng để phục hồi.

\section{TÀI LIỆU THAM KHẢO}

1. Davidson CL, De Gee AJ, "Relaxation of polymerization contraction stresses by flow in dental composites",J Dent Res 1984;63:146-148.

2. Platt JA, Almeida J, Gonzales-Cabezas $C$, Rhodes B, Moore BK, "The effect of double adhesive application on the shear bond strength to dentin of compomers using three one bottle adhesive systems",Oper Dent 2001;26: 313-317.
3. Rajni N, Pallavi $S$, Naveen M (2015), "Influence of double application technique on the bonding effectiveness of self-etch adhesive systems", Microscopy research and technique 78:489-494 (2015)

4. Rueggeberg FA, Margeson DH, "The effect of oxygen inhibition on an unfilled/filled composite

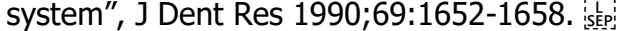

5. Simone deliperi, David N. Bardwell, "Microleakage of a microhybrid composite resin using three different adhesive placement techniques",] adhes Dent

6. Van Meerbeek B, Vargas M, Inoue S, Yoshida Y, Peumans M, Lambrechts P, Vanherle G, "Ádhesives and cements to promote preservation dentistry",Oper Dent 2001;(suppl 6):119-144.

\section{NGHIÊN CỨU MộT Số KÍCH THƯớC ổ CốI, GÓC NGHIÊNG TRƯớC CỦA Cổ XƯƠ'NG ĐÙI VÀ GÓC NGHIÊNG KẾT HỢP TRÊN PHIM CHỤP CẮT LỚP VI TÍNH}

\section{TÓM TẮT}

Qua nghiên cứu trên 54 phim chụp cắt lớp vi tính của 54 bệnh nhân không có bệnh lý khớp háng ở ít nhất 1 bên tại Bệnh viện Đại học Y Hà Nội, chúng tôi thu được kết quả như sau: Giá trị góc nghiêng ngoài ổ cối là: $37.48 \pm 4.95^{\circ}$; Giá trị góc nghiêng trước ổ cối là: $17.2 \pm 5.81^{\circ}$; Giá trị góc nghiêng trước của cổ xương đùi là: $12.03 \pm 7.32^{\circ}$; Giá trị góc nghiêng kết hợp là: $29.23 \pm 9.07^{\circ}$

Từ khóa: Ổ cối, góc nghiêng trước cổ xương đùi, góc nghiêng kết hợp.

\section{SUMMARY}

\section{RESEARCH ON ACETABULLAR PARAMETERS, FEMORAL AND COMBINED ANTEVERSION ANGLES ON COMPUTED- TOMOGRAPHY SCAN}

54 computed-tomography scans of 54 Vietnamese adults with at least 1 non-pathological hip at Hanoi Medical University Hospital are used on our research. The results are: Acetabullar inclination angle: $37.48 \pm$ 4.95; Acetabullar anteversion angle: $17.2 \pm 5.81^{\circ}$; Femoral anteversion angle: $12.03 \pm 7.32^{\circ}$; Combined anteversion: $29.23 \pm 9.07^{\circ}$.

Keyword: Acetabulum, femoral anteversion, combined anteversion.

\footnotetext{
${ }^{1}$ Trường Đại học Y Hà Nội

²Bênh viện Đại học Y Hà Nội

Chịu trách nhiệm chính: Trần Lê Đình Duy

Email: tranledinhduy.hmu@gmail.com

Ngày nhận bài: 7/3/2021

Ngày phản biện khoa học: 9/4/2021

Ngày duyệt bài: 5/5/2021
}

Trần Lê Đình Duy ${ }^{1}$, Trần Sinh Vương ${ }^{1}$, Nguyễn Văn Hoạt ${ }^{2}$, Ngô Xuân Khoa ${ }^{1}$.

\section{I. ĐĂT VẤN ĐỀ}

Những thông tin về các kích thước bình thường của ổ cối, góc nghiêng trước của cổ xương đùi nói riêng và khớp háng nói chung có vai trò rất quan trọng trong việc lựa chọn khớp háng nhân tạo phù hợp với bệnh nhân cũng như giúp phẫu thuật viên dễ dàng đánh giá kết quả hậu phẫuhay khớp háng [1]. Với mục đích cung cấp thêm số liệu bình thường của các kích thước ổ cối và các góc nghiêng trên phim chụp cắt lớp vi tính, đồng thời góp phần giúp các nhà ngoại khoa và các bác sĩ có thêm số liệu tham khảo trong thực hành lâm sàng, chúng tôi tiến hành nghiên cứu với mục tiêu: Khảo sát các kích thước góc nghiêng ngoài ổ cối, góc nghiêng trước ố cối, góc nghiêng trước của cổ xương đưii, góc nghiêng kêt hợp trên phim chụp cắt lớp vi tính ở người Việt Nam trưởng thành.

\section{II. ĐỐI TƯỢNG VÀ PHƯƠNG PHÁP NGHIÊN CỨU}

2.1. Đối tượng nghiên cứu. Phim chụp cắt lớp vi tính qua khung chậu và xương đùi được lưu trữ trong hệ thống cơ sở dữ liệu hình ảnh (Picture Archiving and Communication System PACS) của 54 bệnh nhân độ tuổi từ 25 đến 96 (tuổi trung bình: 61士16.62) không mắc bệnh lý cơ - xương - khớp (viêm khớp, hoại tử chỏm xương đùi, biến dạng khớp háng ...) ở một hoặc cả hai bên khớp tại Khoa Chẩn đoán hình ảnh, Bệnh viện Đại học Y Hà Nội, trong thời gian từ tháng 08/2018 đến tháng 04/2020. 